\title{
Impact of inter-cell interference on capacity in the joint multiple access (CDMA and SDMA) system
}

\begin{abstract}
Spatial filtering using smart antenna has emerged as a promising technique to improve the performance of cellular systems. Cell splitting and sectorisation in CDMA systems could result in an increase in system capacity. In this paper, we investigate the impact of inter-cell interference on reverse link capacity in a joint multiple access system arising from the combination of CDMA and SDMA systems. The system capacity of CDMA and SDMA systems is reviewed individually. The co-channel and antenna side-lobes interferences in SDMA systems due to the randomly located mobile users in a non-uniform traffic cell are studied. Therefore, the most realistic reverse link capacity improvement of the joint multiple access system is presented here by taking into consideration both intra-sector and inter-sector interferences. The results are based on the system parameters of CDMA and SDMA systems.
\end{abstract}

Keyword: CDMA; Inter-cell interference; SDMA; System capacity 